Vol. 6, No. 3-4, 2020

Volodymyr Zelenyak ${ }^{1}$, Liubov Kolyasa ${ }^{2}$, Myroslava Klapchuk ${ }^{3}$

1. Department of Mathematics, Lviv Polytechnic National University, 12, S. Bandery Str., Lviv, Ukraine, e-mail: volodymyr.zelenyak@gmail.com, ORCID 0000-0002-6653-4326

2. Department of Mathematics, Lviv Polytechnic National University, 12, S. Bandery Str., Lviv, Ukraine,

E-mail: kolyasa.lubov@gmail.com, ORCID 0000-0002-9690-8042

2. Department of Mathematics, Lviv Polytechnic National University, 12, S. Bandery Str., Lviv, Ukraine,

E-mail: m.klapchuk @gmail.com, ORCID 0000-0003-4826-0824

\title{
MATHEMATICAL MODELING OF ELASTIC STATE IN A THREE-COMPONENT PLATE CONTAINING A CRACK DUE TO THE ACTION OF UNIDIRECTIONAL TENSION
}

Received: September 12, 2020 / Revised: November 25, 2020 / Accepted: December 30, 2020

(C) Zelenyak V., Kolyasa L., Klaphuk M., 2020

\begin{abstract}
Purpose. A two-dimensional mathematical model for the problem of elasticity theory in a three-component plate containing rectilinear crack due to the action of mechanical efforts is examined. As a consequence, the intensity of stresses in the vicinity of tops of the crack increases, which significantly affects strength of the body. This may lead to the growth of a crack and to the local destruction of a structure. Such a model represents to some extent a mechanism of destruction of the elements of engineering structures with cracks, we determined stress intensity factors (SIFs) at the tops of the crack, which are subsequently used to determine critical values of the tension. Therefore, the aim of present work is to determine the two-dimensional elastic state in plate containing an elastic two-component circular inclusion and crack under conditions of power load in the case of unidirectional tension of the plate perpendicular for the crack line. This makes it possible to determine the critical values of unidirectional tension in order to prevent crack growth, which will not allow the local destruction of the body. Methodology. The methods of studying two-dimensional elastic state body with crack as stress concentrators based on the function of complex variable method by which the problem of elasticity theory is reduced to singular integral equations (SIE) of the first and second kind, the numerical solution by the method of mechanical quadratures was obtained. Findings. In this paper two-dimensional mathematical model in the form of the system of two singular integral equations on closed contour (boundary of inclusion) and unclosed contour (crack) are obtained; numerical solutions of these integral equations were received by the method of mechanical quadratures; stress intensity factors at the tops of a crack are identify and explored to detect the effects of mechanical character. Graphical dependencies of SIFs, which characterize distribution of the intensity of stresses at the tops of a crack as function of elastic properties of inclusion and also as function of the distance between crack and inclusion are obtained. This makes it possible to analyze the intensity of stresses in the vicinity of a crack's tops depending on the geometrical and mechanical factors, as well as to determine the limit of permissible values of unidirectional tension of the plate perpendicular to the crack line at which the crack begins to grow and the body being locally destroyed. It is shown that the proper selection of elastic characteristics of the components of three-component plate can help achieve an improvement in the strength of the body in terms of the mechanics of destruction by reducing SIFs at the crack's tops. Originality. Scientific novelty lies in the fact that the solutions of the new two-dimensional problems of elasticity for a specified region (plate containing an elastic two- component circular inclusion and a rectilinear crack) under the action of unidirectional tension of the plate perpendicular to the crack line are obtained. Practical value. Practical value of the present work lies in the possibility of a more
\end{abstract}




\section{Mathematical Modeling of Elastic State in a Three-Component Plate Containing a Crack ...}

complete accounting of actual stressed-strained state in the piecewise-homogeneous elements of a structure with cracks that work under conditions of different mechanical loads. The results of specific studies that are given in the form of graphs could be used when designing rational operational modes of structural elements. In this case, the possibility for preventing the growth of a crack through the appropriate selection of composite's components with the corresponding mechanical characteristics is obtained.

Keywords: crack, stress intensity factors, singular integral equation, unidirectional tension, three-component plate.

\section{Introduction and literature review}

The study of the stress-strain state of piecewise homogeneous bodies with cracks has not only scientific but also practical significance for assessing the strength and reliability of many elements of engineering structures. Mechanical stresses that occur in the body under the action of external force loads can contribute to the formation of new and the growth of existing cracks and as a result lead to local or complete destruction of the structure or its elements. Therefore, the study of stress distribution in the vicinity of crack vertices is important for calculations of the strength and durability of structural elements, as well as in the development of experimental methods for estimating the resistance of the material to crack propagation.

Elastic interaction between two spherical cracks, located along the outer surface of hollow parts, placed in a heterogeneous environment during action of an uniaxial stretching load was analyzed in [1].

Authors of [2] obtained analytical solution to a two-dimensional problem of elasticity theory of screw dislocation near the surface crack of mode III, a shear crack during action of anti-flat deformation. They determined effect of the dislocation on a stress intensity coefficient.

In paper [3], a problem of interaction between a crack and an elastic inclusion was reduced to solving singular integral equations of the Cauchy type. Based on this result, the authors analyzed singular behavior of the solution for a crack with branches.

A two-dimensional model of a problem on bending a plate weakened by coaxial crack and slit were considered in [4]. The resulting solution makes it possible to analyze the effect of the interaction of variable-type defects on the stressed state near tops.

Based on the numerical solution to the bound three-dimensional elastic-dynamic problem, the influence of massive inclusion of the hard disk on the adjacent slit-like crack was examined in [5].

A problem on the circular, absolutely rigid, inclusion of arbitrary shape, which is located in the transversally isotropic half-space under conditions of smooth contact with the second half-space, was reduced to a system of two-dimensional singular integral equations. Authors of [6] investigated the asymptotes of stresses in the vicinity of an inclusion and defined directions of the largest and the lowest concentration of stresses.

In article [7], a problem of elasticity theory for a half-plane with many cracks was reduced to a singular integral equation using the modified comprehensive potential under condition of free stretching. The authors obtained a system of singular integral equations with a distributed dislocation function.

The method of singular integral equations which was used also for the analysis of the plane elastic state in a piecewise-homogeneous two-dimensional solids with cracks is addressed in a number of publications. In particular, a crack was studied, located in parallel to the lines of junction of dissimilar halfplanes when stretching stresses are assigned on the infinity of the formed plane [8]. Termoelastic problem for such a region with uniform distribution of temperature over entire piecewise-homogeneous plane with a crack was examined in [9].

An analysis of major scientific literary sources revealed that still unexplored and undeveloped are the mathematical models, which are applied to study the interaction between a crack and the line that connects two dissimilar half-planes, as well as the intersection of a crack with the line of junction in piecewise homogeneous bodies with cracks. Given this, there is a necessity to construct mathematical 


\section{Volodymyr Zelenyak, Liubov Kolyasa, Myroslava Klapchuk}

models for determining such mechanical loads at which a crack starts to grow while the body undergoes local destruction. Exploring such models will make it possible to propose one of the approaches, for example by selecting the components of piecewise homogeneous bodies, welded together, with appropriate mechanical characteristics, to prevent the growth of a crack.

This model is the development of the previous models to determine the two-dimensional elastic state in a three-component piecewise-homogeneous solid, weakened by a crack.

\section{Statement of the problem}

Let the infinite plate consist of an elastic matrix $S$ and two dissimilar elastic isotropic inclusions $S_{0}$ and $S_{1}$ bounded by smooth contours $L_{0}$ and $L_{1}$, accordingly, where $S_{0}$ is a circular inclusion (disk) of radius $R_{0}, S_{1}$ is a circular ring with an inner radius $R_{0}$ and an outer radius $R_{1}$ with a center at the origin $x O y$. The matrix contains rectilinear crack $L_{2}$ of length $2 l$ located at the axis and the center of the crack is at the point $(d, 0)$. At infinity, the plate is stretched by external stresses $p$ directed perpendicular to the crack line $L_{2}$ (Fig. 1).

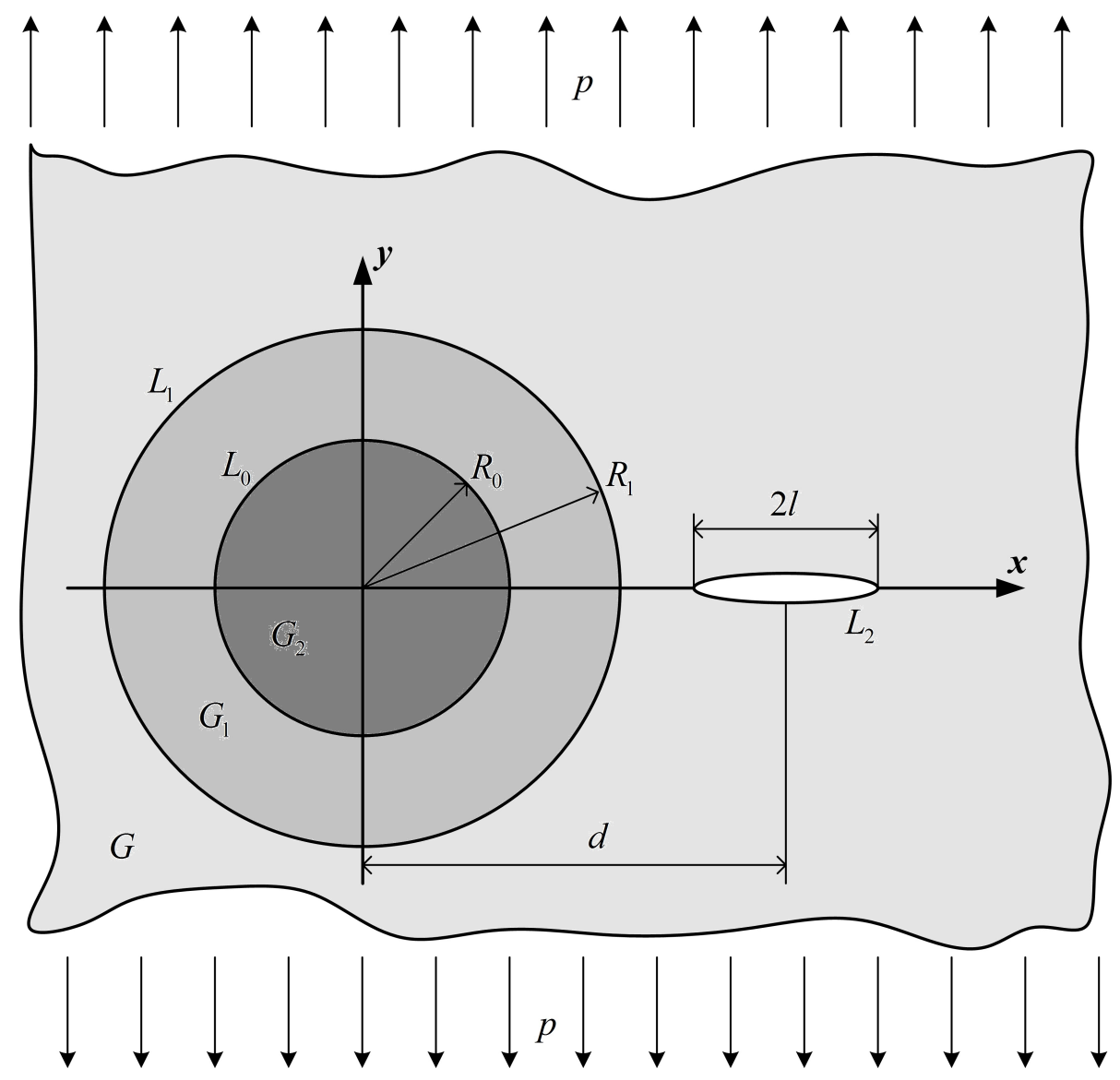

Fig. 1. Tensile plate with two-component folded circular inclusion and crack

Assume that the contour of the ring $L_{1}$ and the contour of the disk $L_{0}$ is set to the ideal-mechanical contact (equality of stresses and displacements when approaching the left and right to the circuit):

$$
\begin{aligned}
& {\left[N\left(t_{1}\right)+i T\left(t_{1}\right)\right]^{+}=\left[N\left(t_{1}\right)+i T\left(t_{1}\right)\right]^{-} ;} \\
& \left(u_{1}+i v_{1}\right)^{+}-\left(u_{1}+i v_{1}\right)^{-}=0, \quad t_{1} \in L_{1} ; \\
& {\left[N\left(t_{0}\right)+i T\left(t_{0}\right)\right]^{+}=\left[N\left(t_{0}\right)+i T\left(t_{0}\right)\right]^{-} ;} \\
& \left(u_{0}+i v_{0}\right)^{+}-\left(u_{0}+i v_{0}\right)^{-}=0, \quad t_{0} \in L_{0} .
\end{aligned}
$$


The lips of the crack are not in contact during the deformation process and are free from loads:

$$
\left[N\left(t_{2}\right)+i T\left(t_{2}\right)\right]^{ \pm}=0, \quad t_{2} \in L_{2} .
$$

In relations (3) $N\left(t_{n}\right), T\left(t_{n}\right)$ are normal and tangential stress components, $u_{n}, v_{n}$ are displacement components.

\section{Mathematical model}

Complex stress potentials $\Phi(z), \Psi(z)$ can be represented in the form [10]:

$$
\Phi(z)=\Phi_{0}(z)+\Phi_{1}(z)+\Phi_{2}(z) ; \quad \Psi(z)=\Psi_{0}(z)+\Psi_{1}(z)+\Psi_{2}(z),
$$

where

$$
\begin{aligned}
& \Phi_{1}(z)=\frac{1}{2 \pi} \sum_{k=1}^{2} \int_{L_{k}} \frac{Q_{k}\left(t_{k}\right) d t_{k}}{\zeta_{k}-z} \\
& \Psi_{1}(z)=\frac{1}{2 \pi} \sum_{k=1}^{2} \int_{L_{k}}\left[\frac{\overline{Q_{k}\left(t_{k}\right)} \overline{d t_{k}}}{\zeta_{k}-z}-\frac{\overline{\zeta_{k}} Q_{k}\left(t_{k}\right) d t_{k}}{\left(\zeta_{k}-z\right)^{2}}\right] ; \\
& \zeta_{k}=t_{k}+d \\
& Q_{k}\left(t_{k}\right)=\left\{\begin{array}{c}
g_{1}\left(t_{1}\right), k=1, \\
g_{2}^{\prime}\left(t_{2}\right), k=2 ;
\end{array}\right. \\
& \Phi_{2}(z)=\frac{1-\Gamma_{01}}{2 \pi\left(1+\chi_{1} \Gamma_{01}\right)} \sum_{k=1}^{2} \int_{L_{k}}\left[\frac{R_{0}^{2} Q_{k}\left(t_{k}\right) d t_{k}}{z\left(z \overline{\zeta_{k}}-R_{0}^{2}\right)}+\frac{\left(R_{0}^{2}-\zeta_{k} \overline{\zeta_{k}}\right) \overline{Q_{k}\left(t_{k}\right)} \overline{d t_{k}}}{\overline{\zeta_{k}}\left(R_{0}^{2}-z \overline{\zeta_{k}}\right)^{2}}\right] ;
\end{aligned}
$$

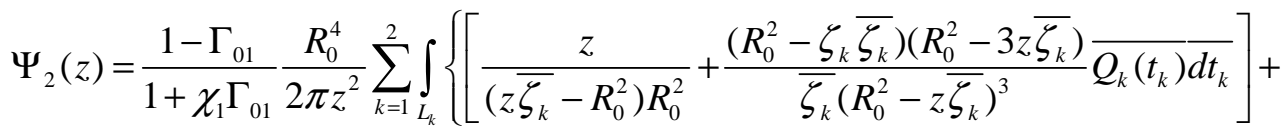

$$
\begin{aligned}
& \left.+\left[\frac{3 z \zeta_{k}-2 R_{0}^{2}}{z\left(z \overline{\zeta_{k}}-R_{0}^{2}\right)^{2}}+\frac{1}{R_{0}^{2} \zeta_{k}}\right] Q_{k}\left(t_{k}\right) d t_{k}\right\},|z|>R_{0},\left|\zeta_{k}\right|>R_{0} ;
\end{aligned}
$$

$g_{1}\left(t_{1}\right)$ is unknown function on the contour $L_{1} ; g_{2}^{\prime}\left(t_{2}\right)$ is unknown derivative of the jump of displacements at the transition through the crack line $L_{2}$. The function $g_{2}^{\prime}\left(t_{2}\right)$ must have integrative features at the ends of the crack. $\Phi_{0}(z)=p / 4, \Psi_{0}(z)=p / 2$ are the potentials that determine the stress state of a homogeneous plane without cracks under the action of tension at infinity by external stresses $p$ [10]; $\Gamma_{01}=G_{0} / G_{1} ; G_{0}\left(G_{1}\right)$ is disk (ring) displacement module. Complex potentials $\Phi_{1}(z), \Psi_{1}(z)$, $\Phi_{2}(z), \Psi_{2}(z)$ characterize the perturbed stress state due to the two-component inclusion and crack.

Note that the choice of complex potentials in the form of (4), (5) provides exact satisfaction of the second equality of the boundary condition (2) on the circuit $L_{0}$. As a result, the order of the system of integral equations obtained after satisfying the other boundary conditions is reduced from three to two.

Satisfying with the use of complex potentials (4), (5) the second equality of the boundary condition (1) on the contour $L_{1}$ and the boundary condition (3) on the crack $L_{2}$, we obtain the system of two singular integral equations of the second and first kind, respectively, with respect to two unknown functions $Q_{1}\left(t_{1}\right)$ on the contour $L_{1}$ and $Q_{2}\left(t_{2}\right)$ on the contour $L_{2}$ :

$$
\begin{gathered}
A_{1} Q_{1}\left(\tau_{1}\right)+\frac{1}{2 \pi} \int_{L_{1}}\left[R_{11}\left(t_{1}, \tau_{1}\right) Q_{1}\left(t_{1}\right) d t_{1}+S_{11}\left(t_{1}, \tau_{1}\right) \overline{Q_{1}\left(t_{1}\right)} \overline{d t_{1}}\right]+ \\
+\frac{1}{2 \pi} \int_{L_{2}}\left[R_{12}\left(t_{2}, \tau_{1}\right) Q_{2}\left(t_{2}\right) d t_{2}+S_{12}\left(t_{2}, \tau_{1}\right) \overline{Q_{2}\left(t_{2}\right)} \overline{d t_{2}}\right]=P_{1}\left(\tau_{1}\right), \quad \tau_{1} \in L_{1} ; \\
\frac{1}{2 \pi} \int_{L_{1}}\left[R_{21}\left(t_{1}, \tau_{2}\right) Q_{1}\left(t_{1}\right) d t_{1}+S_{21}\left(t_{1}, \tau_{2}\right) \overline{Q_{1}\left(t_{1}\right)} \overline{d t_{1}}\right]+ \\
+\frac{1}{2 \pi} \int_{L_{2}}\left[R_{22}\left(t_{2}, \tau_{2}\right) Q_{2}\left(t_{2}\right) d t_{2}+S_{22}\left(t_{2}, \tau_{2}\right) \overline{Q_{2}\left(t_{2}\right)} \overline{d t_{2}}\right]=P_{2}\left(\tau_{2}\right), \quad \tau_{2} \in L_{2},
\end{gathered}
$$


where

$$
\begin{aligned}
& R_{n k}\left(t_{k}, \tau_{n}\right)=R_{n k}^{1}\left(t_{k}, \tau_{n}\right)+R_{0}^{2} \frac{1-\Gamma_{01}}{1+\chi_{1} \Gamma_{01}}\left\{\frac{B_{n}}{\eta_{n}\left(\eta_{n} \bar{\zeta}_{k}-R_{0}^{2}\right)}-C_{n}\left\{\frac{R_{0}^{2}-\zeta_{k} \overline{\zeta_{k}}}{\zeta_{k}\left(R_{0}^{2}-\overline{\eta_{n}} \zeta_{k}\right)^{2}}+\frac{\overline{d \tau_{n}}}{d \tau_{n}}\left[\frac{1}{\eta_{n}\left(\overline{\eta_{n}} \zeta_{k}-R_{0}^{2}\right)}+\right.\right.\right. \\
& \left.\left.\left.+\frac{\left[R_{0}^{2}\left(R_{0}^{2}-3 \overline{\eta_{n}} \zeta_{k}\right)+2 \eta_{n} \zeta_{k}\left(\overline{\eta_{n}}\right)^{2}\right]\left(\zeta_{k} \overline{\zeta_{k}}-R_{0}^{2}\right)}{\zeta_{k}\left(\overline{\eta_{n}}\right)^{2}\left(\overline{\eta_{n}} \zeta_{k}-R_{0}^{2}\right)^{3}}\right]\right\}\right\} \\
& S_{n k}\left(t_{k}, \tau_{n}\right)=S_{n k}^{1}\left(t_{n}, \tau_{n}\right)+R_{0}^{2} \frac{1-\Gamma_{01}}{1+\chi_{1} \Gamma_{01}}\left\{\frac{B_{n}\left(R_{0}^{2}-\zeta_{k} \overline{\zeta_{k}}\right)}{\zeta_{k}\left(R_{0}^{2}-\eta_{n} \overline{\zeta_{k}}\right)^{2}}-\left\{C_{n} \frac{1}{\overline{\eta_{n}}\left(\overline{\eta_{n}} \zeta_{k}-R_{0}^{2}\right)}+\right.\right. \\
& \left.\left.+\left[\frac{\overline{d \tau_{n}}}{d \tau_{n}} \frac{1}{\overline{\zeta_{k}}\left(\overline{\eta_{n}}\right)^{2}}+\frac{\eta_{n} \overline{\eta_{n}}\left(R_{0}^{2}-2 \overline{\eta_{n}} \zeta_{k}\right)+3 \overline{\eta_{n}} \zeta_{k} R_{0}^{2}-2 R_{0}^{4}}{\left(\overline{\eta_{n}}\right)^{3}\left(R_{0}^{2}-\overline{\eta_{n}} \zeta_{k}\right)^{2}}\right]\right\}\right\} \\
& R_{n k}^{1}\left(t_{k}, \tau_{n}\right)=\frac{B_{n}}{\zeta_{k}-\eta_{n}}-\frac{C_{n}}{\overline{\zeta_{k}}-\overline{\eta_{n}}} \frac{\overline{d \tau_{n}}}{d \tau_{n}} \\
& S_{n k}^{1}\left(t_{k}, \tau_{n}\right)=-C_{n}\left[\frac{1}{\overline{\zeta_{k}}-\overline{\eta_{n}}}-\frac{\overline{d \tau_{n}}}{d \tau_{n}} \frac{\zeta_{k}}{\left(\overline{\zeta_{k}}-\overline{\eta_{n}}\right)^{2}}\right], \eta_{n}=\tau_{n} e^{i \alpha_{n}}+z_{n}^{0} ; \quad n=1,2, \quad k=1,2 ; \\
& A_{1}=i\left[1+\chi_{1}+\Gamma_{1}(1+\chi)\right] / 2 ; \quad \Gamma_{1}=G_{1} / G ; \quad \delta_{n}=\left\{\begin{array}{c}
1, n=1 \\
0, n=2
\end{array} ;\right. \\
& B_{n}=\left(\chi_{1}-\Gamma_{1} \chi-1\right) \delta_{n}+1 ; \quad C_{n}=\left(2-\Gamma_{1}\right) \delta_{n}-1 ; \\
& P_{n}\left(\tau_{n}\right)=\delta_{n}\left\{-B_{n} \Phi_{0}\left(\eta_{n}\right)+C_{n}\left[\overline{\Phi_{0}\left(\eta_{n}\right)}+\frac{\overline{d \tau_{n}}}{d \tau_{n}}\left(\eta_{n} \overline{\Phi_{0}^{\prime}\left(\eta_{n}\right)}+\overline{\Psi_{0}\left(\eta_{n}\right)}\right)\right]+2 G_{n} g_{n}^{*}\left(\eta_{n}\right)\right\}+ \\
& +\left(1-\delta_{n}\right)\left\{p_{n}^{*}\left(\eta_{n}\right)-\Phi_{0}\left(\eta_{n}\right)-\overline{\Phi_{0}\left(\eta_{n}\right)}-\left[\eta_{n} \overline{\Phi_{0}^{\prime}\left(\eta_{n}\right)}+\overline{\Psi_{0}\left(\eta_{n}\right)}\right]\right\}, \quad n=1,2 ; \quad \eta_{n}=\tau_{n}+d .
\end{aligned}
$$

The system of integral equations (5.16) with an arbitrary right-hand side has a unique solution under the condition:

$$
\int_{L_{2}} g_{2}^{\prime}\left(t_{2}\right) d t_{2}=0
$$

which provides unambiguous movements by passing the crack contour.

Then the stress intensity factors (SIFs) $K_{I}$ and $K_{I I}$, which are the real quantities that characterize the stress-deformed state in the vicinity of the crack tips, are found according to the formula [10]:

$$
K_{I}^{ \pm}-i K_{I I}^{ \pm}= \pm \lim _{t_{2} \rightarrow \pm l_{2}} \sqrt{2 \pi\left|t_{2} \mp l_{2}\right|} Q_{2}\left(t_{2}\right) .
$$

The numerical solution of the system equations (6), (7), are found by the method of mechanical quadrature [10].

\section{Numerical analysis}

Graphs for dimensionless stress intensity factors (SIFs) $F_{1}^{ \pm}=K_{I}^{ \pm} / p \sqrt{l} \quad\left(F_{2}^{ \pm}=0\right)$ are shown in Figs. 2-4. The solid curves correspond to the SIF $F_{1}^{-}$at the left top of the crack $x_{2}=-l$ (closest to the ring), dashed curves correspond to the SIF $F_{1}^{+}$at the right top $x_{2}=l$ (far to the ring). Numerical solutions of the system of singular integral equations (6), (7) are obtained by the method of mechanical quadratures for different values of elastic and geometric parameters of the plate, when $l / R_{0}=0.5 ; d / R_{0}=2$; $R_{1} / R_{0}=1.1 ; \chi_{0}=\chi_{1}=\chi=2$.

In Figs. 2 and 4, curves 1 correspond to the value $G_{1} / G=0.5 ; 2-G_{1} / G=1 ; 3-G_{1} / G=2$; in Fig. 3 curves 1 correspond to the value $G_{0} / G=0.5 ; 2-G_{0} / G=1 ; 3-G_{0} / G=2$. 
Increasing the stiffness of the disk (shear modulus) causes an increase of SIF $F_{1}^{ \pm}, F_{1}^{ \pm}$regardless of the rigidity of the inclusion. In this case, the increase in the stiffness of the inclusion slows down this decrease of SIF $F_{1}^{-}$(Fig. 3). As the crack is removed, the SIF $F_{1}^{ \pm}$decreases at both crack vertices (Fig. 3). Increasing the stiffness of the disk (shear module $G_{0}$ ) causes an increase of SIF $F_{1}^{ \pm}, F_{1}^{ \pm}$regardless of the rigidity of the inclusion. In this case, the increase in the stiffness of the inclusion slows down this decrease of SIF $F_{1}^{-}$(Fig. 3). With the removal of the crack SIF $F_{1}^{ \pm}$decreases in both vertices of the crack (Fig. 4).

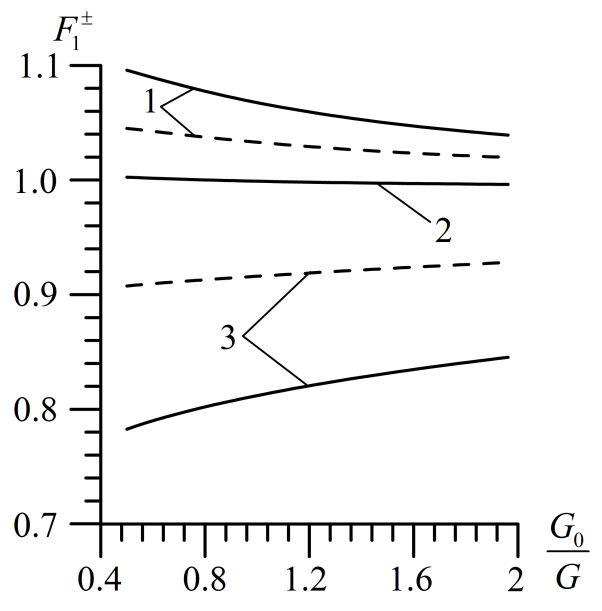

Fig. 2. Dependencies of SIF $F_{1}^{ \pm}$on the relative hardness of the disk $G_{0} / G$

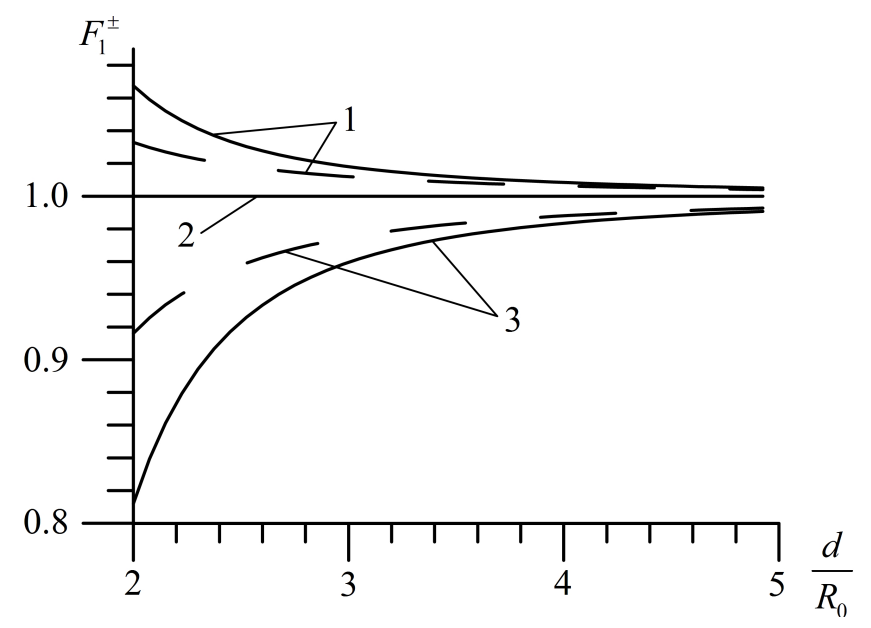

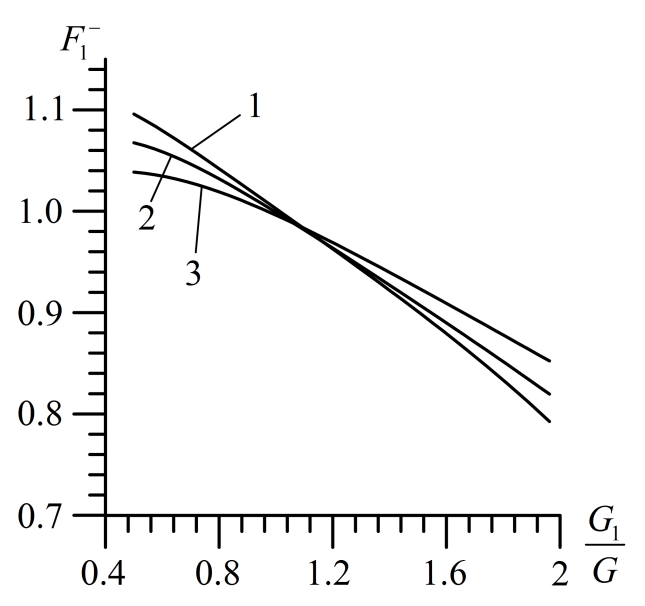

Fig. 3. Dependencies of SIF $F_{1}^{ \pm}$on the relative hardness of the ring $G_{1} / G$

Fig. 4. Dependencies of SIF $F_{1}^{ \pm}$on the relative distance between the centers of inclusion and crack

In all the above cases SIF $F_{1}^{-}$at the top of the crack closest to the ring is larger (smaller) than in the distant SIF $F_{1}^{+}$, if the stiffness of the ring is less (greater) than the matrix.

In the considered problem, the lips of the crack are not in contact. Then, according to the criterion (based on the hypothesis of the initial growth of the crack) from equations of the boundary equilibrium [11] it is possible to find the critical values of the unidirectional tension of the plate perpendicular for the crack line $p_{c r}$, at which the growth of the crack and the local destruction of the body begin, according to the following formula:

$$
p_{c r}=\frac{K_{1 C}}{F_{1}^{ \pm}}
$$

where $K_{1 C}$ is a constant of the material that characterizes the resistance of the material to the destruction and is determined experimentally. 


\section{Volodymyr Zelenyak, Liubov Kolyasa, Myroslava Klapchuk}

Based on the analysis of numerical results for SIF $F_{1}^{ \pm}$from formula (9) it follows: with increasing tensile external stresses $p$, directed perpendicular to the crack line, crack growth will start from the right vertex (far to the ring) if the ring is stiffer than the matrix $\left(G_{1}>G\right)$. In this case $p_{c r}$, is greater than $K_{1 C}$ for both crack vertices. If the ring is less rigid $\left(G_{1}<G\right)$, then vice versa from the left vertex (closest to the ring) for different disk stiffnesses (Fig. 2). In this case $p_{c r}$, is less than $K_{1 C}$ for both crack vertices.

\section{Conclusions}

1. A two-dimensional mathematical model for the problem of elasticity theory for three-component plate containing crack under the action of power load in the form of system of singular integral equations (SIE) of the first and second kind on the contours of crack and inclusion are constructed. Such an approach makes it possible to obtain a numerical solution to SIE by the application of the method of mechanical quadratures.

2. Numerical solutions to SIE (employing the method of mechanical quadratures) in particular case of unidirectional tension of the plate perpendicular to the crack line are obtained. This makes it possible to determine stress intensity factors (SIFs) at the tops of the crack, which are subsequently used to determine critical values of the tension at which a crack starts to grow.

3. Graphic dependencies of SIFs $F_{1}^{ \pm}$, which characterize the distribution of intensity of stresses at the tops of a crack, on the elastic characteristics of three-component plate are constructed. These results make it possible to determine the limit of permissible values of unidirectional tension and could be used when designing rational operational modes of structures' elements in terms of preventing the growth of cracks.

\section{References}

[1] N. Gupta, G. Tagliavia, M. Porfiri, "Elastic interaction of interfacial spherical-cap cracks in hollow particle filled composites", International Journal of Solids and Structures, vol. 48, issues 7-8, pp. 1141-1153, 2011.

[2] S. N. G. Chu, "Elastic interaction between a screw dislocation and surface crack", Journal of Applied Physics, vol. 53, issue 12, pp. 8678-8685, 1982.

[3] Z. Ming-Huan, T. Renji, "Interaction between crack and elastic inclusion", Applied Mathematics and Mechanics, vol. 16, issue 4, pp. 307-318, 1995.

[4] I. P. Shatskyi, T. M. Daliak, "Vzaiemodiya trishchyny z kolinearnoiu shchilynoiu za zghynu plastyny" ["Interaction of the crack with the collinear slit by bending the plate"], Visnyk Zaporizkoho natsionalnoho universytetu. Fizyko-matematychni nauky [Bulletin of Zaporizhzhya National University. Physical and mathematical sciences], vol. 1, pp. 211-218, 2015. [in Ukrainian].

[5] V. V. Mykhas'kiv, O. M. Khay, "Interaction between rigid-disc inclusion and penny-shaped crack under elastic time-harmonic wave incidence", International Journal of Solids and Structures, vol. 46, issue 3-4, pp. 602-616, 2009.

[6] O.F. Kryvyy, "Interface circular inclusion under mixed conditions of interaction with a piecewise homogeneous transversally isotropic space”, Journal of Mathematical Sciences, vol. 184, issue 1, pp. 101-109, 2012.

[7] N. R. F. Elfakhakhre, N. M. A. Nik Long, Z. K. Eshkuvatov, "Stress intensity factor for multiple cracks in half plane elasticity”, AIP Conference Proceedings, vol. 1795, Article ID 020010, 2017.

[8] V. Zeleniak, R. Martyniak, B. Slobodian, "Napruzhennia v spaianykh riznoridnykh pivploshchynakh z vkliuchenniam i trishchynoiu za dii roztiahu" ["Stress in soldered heterogeneous half-planes with inclusion and crack under the action of tension”], Visnyk Natsionalnoho universytetu «Lvivska politekhnika» [Bulletin of Lviv Polytechnic National University], vol. 625, pp. 54-58, 2008. [in Ukrainian].

[9] V. Zeleniak, B. Slobodian, "Modeliuvannia termopruzhnoho dvovymirnoho stanu dvokh spaianykh riznoridnykh pivploshchyn z vkliuchenniamy i trishchynamy" ["Modelling of thermoelastic two-dimensional state of bonded heterogeneous halfplanes with inclusions and cracks"], Fizyko-matematychne modeliuvannia ta informatsiyni tekhnolohiy [Physico-mathematical modeling and informational technologies], vol. 12, pp. 94-101, 2010. [in Ukrainian].

[10] M. P. Savruk, Dvumernye zadachi uprugosti dlya tel s treshchinami [Two-dimensional elasticity problems for bodies with cracks]. Kyiv, Ukraine: Naukova dumka Publ., 1981. [in Russian].

[11] V. V. Panasyuk, M. P. Savruk, A. P. Datsyshin, Raspredelenie napryazheniy okolo treshchin v plastinah $i$ obolochkah [Distribution of stresses near cracks in plates and shells]. Kyiv, Ukraine: Naukova dumka Publ., 1976. [in Russian]. 